\title{
Optimization Algorithm of Holes Machining Path
}

\author{
Jing Zhang ${ }^{1, \text { a }}$ \\ ${ }^{1}$ Basic Courses Department, Beijing Union University, Beijing, China \\ azhang1jing4@sina.com
}

Keywords: traveling salesman problem, path planning, genetic algorithm, group holes machining

\begin{abstract}
Holes machining path planning is of great significance to improve the efficiency and quality of the processing of porous parts. Hole group on the characteristics of size, shape, and location of different multi-bit processing optimization and process optimization, a reasonable choice of the machining path planning method. Verified by an example of this optimization method significantly improved in the holes machining $\mathrm{CNC}$ machining efficiency, reduce processing costs. Holes machining path optimization analysis and description of the problem, to clear this problem are essentially multi-point optimization method and process optimization, and the optimization method to determine the selection principle.
\end{abstract}

\section{Introduction}

People tend to focus on how to improve the processing time for each hole, to the neglect of the tool shift, tool change and other auxiliary time. In fact, the auxiliary time holes machining productivity is greater than the processing time, especially when to be a huge amount of processing hole, especially. The survey shows that the average occupancy of $70 \%$ of the total processing time in the movement of the tool and the workpiece during machining. The hole diameter, depth, errors and surface roughness of the base requirements, you need a large number of different tools and tool change operation, a considerable amount of time spent on tool transposition processing and tool change operation. Therefore, how to plan the holes machining path group, in order to shorten the tool mobile path, reducing the number of tool changes and tool change times, holes machining of key issues.

However, in generating the NC instruction, the existing PCB automatic programming software used by hole position of the $\mathrm{X}, \mathrm{Y}$ coordinates to an agreed method of successive arrangement to determine drilling pass sequence. Obviously, the thus generated drilling cutting line is not the optimum route, affect the production efficiency. For those thousands of pieces of annual production, mass production scale to tens of ten thousand PCB dedicated manufacturers, the extent of its impact is considerable. In order to improve processing efficiency, the drilling tool path planning is a very important issue.

\section{Background to the issue}

Vias is one of the important parts of the printed circuit boards (also referred to as printed circuit board), the processing cost of the vias typically accounts for $30 \%$ to $40 \%$ of the cost of the system board, and drilling machines are mainly used in the PCB manufacturing process in the punching operation. The questions are designed to improve the performance of the production of certain types of Punch.

Punch production performance depends mainly on the following aspects: (1) a single hole drilling operations time, which is determined by the production process To simplify the problem, assume that the time for the same hole drilling operations are the same; (2) of the drilling machine in the machining operation, the drill advancing time; (3) for different grooved machining operation, the tool conversion time. At present, the actual use of the drilling machine is generally a single drill job, i.e. a drill bit for drilling.

Existing certain drill, the tool is equipped with eight kinds of above a, b, c, .., h, sequentially arranged in a circular annular. And 8 kinds of tools in the order fixed and can not be swapped. In the 
machining operation, a tool after use can be converted to use another tool. Two adjacent tool conversion times is $18 \mathrm{~s}$, for example, converted to the cutter B used by the tool a time is $18 \mathrm{~s}$, the other case and so on. The way to convert the tool operation, clockwise rotation can be used, for example, converted to the tool the b; may also be used counterclockwise manner from the tool a conversion tool, for example, from the tool a conversion to the cutter $\mathrm{h}$. But in the same circuit board through-hole does not require the completion of processing a hole, and then processing the another hole, i.e. for the vias to be processed by two or more tool, as long as the required tooling order correct button. In order to improve the performance of the drilling machine, it is now to design a dual-drill drilling machine (each shape with a single drill bit of the drill is the same), the two drill can be operating simultaneously, and the operations are independent, which can be two of the drill bit for drilling can also drill holes in the road or conversion tool of another drill. To avoid touching between the drill bit and interference processing at any time in the vias must maintain the two drill spacing of not less than $3 \mathrm{~cm}$ (called the two drill cooperation spacing). In order to simplify the problem, the drill can be seen as a particle.

\section{Mathematical Modeling}

A total of $n$ holes, $i, j$ hole spacing $d_{i j}$, drill traveling unit cost is $a$, Traveling speed is $v$, the drill travel costs for:

$$
f=\sum_{i, j=1, i \neq j}^{n} a d_{i j} e_{i j}
$$

The drill advancing time is

$$
t_{f}=\sum_{i, j=1, i \neq j}^{n} \frac{d_{i j} e_{i j}}{v}
$$

where $e_{i j}= \begin{cases}1, & i, j \text { on the travel path, } \\ 0, & \text { otherwise. }\end{cases}$

$i, j$ between two holes tool changer time $p_{i j}$, tool replacement unit cost is $b$, the tool replacement costs is:

$$
g=\sum_{i, j=1, i \neq j}^{n} b p_{i j} e_{i j} \text {. }
$$

Tool change time is:

$t_{g}=\sum_{i, j=1, i \neq j}^{n} p_{i j} e_{i j}$

where $e_{i j}= \begin{cases}1, & i, j \text { on the travel path, } \\ 0, & \text { otherwise. }\end{cases}$

\section{Objective function}

Normal circumstances, seeking the production time, the economic costs are minimum operating program, namely:

$$
\begin{gathered}
\min f^{(1)}+g^{(1)}+f^{(2)}+g^{(2)} \\
\min \max \left\{t_{f}^{(1)}+t_{g}^{(1)}, t_{f}^{(2)}+t_{g}^{(2)}\right\}
\end{gathered}
$$

wherein (1), (2) represents the 1 st bit and No. 2 of the drill. Further, since the relationship between the two drills bits also need some constraint conditions.

However, in extreme circumstances, such as in short supply, more consideration to the production time to be small; when supply exceeds demand, to consider the cost as much as possible.

\section{Greedy algorithm}


TSP problem is a complex problem in the practical application centralized summarized and simplified form, is a simple concept, but to solve complex problems, solving the task is to arrange a complete travel, so that the total minimum cost, the solving process is a search processn city TSP problem search space is a collection of all permutations of $n$ cities, the size of $n$ ! TSP problem most intuitive greedy algorithm is based on the field of heuristic methods: random from a city view, go to the nearest city not access until all cities have been visited and are accessed only once, lastinitial return to the city, such a path is difficult to be perfect, and usually pay a high price for the greedy choice in the initial stage.

The algorithm solving process:

(1) Encoding and established solution space (with the genetic algorithm).

(2) Find the edge weight matrix. Seeking the distance between any two points, as the edge weight between two points. Corresponding weights stored in the matrix $M$, its line marked the starting point; the column marked the end obviously $M$ symmetric matrix.

(3) Find the nearest neighbors. Let $k=n$ in $M$ worth to find the minimum weight edge $e_{i j}$, processing the path loop point into and out of degrees are 1 , so get rid of the $i$-th row and $j$ column, get $M_{0}$ while preserving edges $e_{i j}$, set $k=k-1$.

(4) End of the algorithm conditions when $k=1$, the end of the program, while preserving the edge in $M$.

\section{Genetic Algorithms}

Genetic algorithm to optimize holes machining path first need to construct chromosome, chromosome length generally equal to the number of holes. Direct coding such as the number of the holes, some holes are not processed after the crossover or mutation may occur. The operation of the genetic algorithm, copy, crossover, and mutation operation is evolving populations, the solution of the problem is an important step gradually toward optimal. Traveling salesman path problem solving, chromosome replication is generally used in the method of the best retention roulette copy, two chromosomes cross multi-exchange subsequent gene randomly select a mate; the chromosomal aberrations operation is in certain mutation rate under randomly generated a number of the hole as the first machining holes thus changing the machining path of the hole group. Single drill holes machining path optimization problem to be converted into the traveling salesman problem, and genetic algorithm as a global search ability of the algorithm widely used in solving the TSP problem.

The algorithm solving process:

(1) Encoding used encoding binary representation, neighbors said, the order said, the path matrix representation and the edges represent which path due to natural, intuitive, and easy to join the heuristic information, using up its convention coding way.

(2) To establish the solution space would be a solution in the space is represented as a circular arrangement $a=\left(a_{1}, a_{2}, \ldots, a_{n}\right)$, where $a_{i}(1 \leqq i \leqq \mathrm{n})$. The $i$-th path after the number of the holes, when $i \neq j, a_{i} \neq a_{j}$ a collection of all the solutions constituting the solution space $S$, i.e., $S=\left\{\left(a_{1}, a_{2}, \ldots\right.\right.$, $\left.\left.a_{n}\right)\right\}$, wherein each cycle ordering shows the traverse of a circuit of the $n$-th hole.

(3) To determine the initial population, the number $N$ of chromosome in the initial population to determine, generally based on the number $\mathrm{n}$ of the group of holes in the hole can be set to $N=n / 8$, the composition of the chromosome using the nearest neighbor method is started from the initial position to find until it covers the entire hole group, and ultimately generate $N$ chromosomes.

(4) Determine the selection operator. Genetic algorithm is generally proportional selection operator, that operator is calculated by the following probabilistic rules.

(5) Determine the crossover operator. The multiple crossover operator used in genetic algorithms heuristic crossover and edge recombination crossover relatively effective.

(6) To determine the mutation operator. Currently applied to the genetic algorithm mutation operator: 2-opt mutation operator, inversion mutation operator. Shift mutation operator insertion mutation operator, which is relatively effective heuristic mutation operator. 
(7) Determined that the termination conditions, the following can be used as the termination condition of the algorithm:

$1^{\circ}$ best individual remains unchanged for 20 generations;

$2^{\circ}$ number of iterations $t>8 n$;

$3^{\circ}$ the difference between the population mean fitness of the best individual fitness small in $\xi$ ( $\xi$ is an arbitrarily small positive real number), but in each case the effect of various processing environment, select the termination condition to have the actual decision.

\section{Conclusion}

Effective solution to the printed circuit board carrying the porous machining tool path air travel lengthy low processing efficiency, shortening the printed circuit board the CNC processing time, improve production efficiency. To introduce dual drill at the same time processing is the effective way to improve the efficiency of holes machining, analysis of the double drill hole machining path optimization problem of the objective function and constraints, the modified genetic algorithm for single drill holes machining path optimization for dual drill holes machining path optimization algorithm. One chromosome processing path, the fitness of the two drills requires the length of time said the two-bit processing individual parts. Genetic algorithm optimization ideal path, not easy to fall into local optimization, to make up for the higher its algorithm complexity insufficient Therefore, the algorithm is a very good performance general-purpose global optimization algorithm of this kind of algorithm design prevent algorithm "premature" phenomenon, which can be controlled by setting the mutation probability, high mutation rate of expansion of the search space is conducive to produce more good individuals, crossover and mutation probability over the General Assembly led to the convergence speed too slow, poor control will cause the number of iterations is too large, the program was forced to terminate.

\section{Acknowledgments}

The work is supported by Funding Project for Academic Human Resources Development in Institutions of Higher Learning under the Jurisdiction of Beijing Municipality (PHR (IHLB)) (PHR201108407). Thanks for the help.

\section{References}

[1] Bodo Manthey. Deterministic algorithms for multi-criteria Max-TSP. Discrete Applied Mathematics, v 160, n 15, p 2277-2285, October 2012.

[2] Jing Zhang. Modeling and Solution for Multiple Chinese Postman Problems. Communications in Computer and Information Science, 2011, 215(2): 520-525.

[3] Zhou, Yong-Quan; Huang, Zheng-Xin; Liu, Hong-Xia. Discrete glowworm swarm optimization algorithm for TSP problem. Tien Tzu Hsueh Pao/Acta Electronica Sinica, v 40, n 6, p 1164-1170, June 2012.

[4] Jing Zhang. A New Non-monotone Line Search Algorithm for Nonlinear Programming. Przeglad Elektrotechniczny, 2012, 88(7b): 265-268.

[5] Bazgan, Cristina; Gourvès, Laurent; Monnot, Jérôme; Pascual, Fanny. Single approximation for biobjective max TSP. Lecture Notes in Computer Science, v 7164 LNCS, p 49-62, 2012.

[6] Boyd, Sylvia; Carr, Robert. Finding low cost TSP and 2-matching solutions using certain half-integer subtour vertices. Discrete Optimization, v 8, n 4, p 525-539, November 2011. 